\title{
Symbolic transfer entropy analyzed sleep electroencephalogram
}

\author{
Ruijun Chang, Liu Yang, Qiuping Chen, Jun Wang \\ Image Processing and Image Communications Key Lab, College of Geo \& Bio Infor- \\ mation,Nanjing Univ of Posts \& Telecomm, Nanjing 210003, China \\ wangj@njupt.edu.cn
}

\begin{abstract}
The quality of sleep has a great relationship with health. The result of sleep stage classification is an important indicator to measure the quality of sleep. It was found that the symbolic transfer entropy about the $\alpha$ wave of the wake and the first stage of non-rapid eye movement sleep reflect on the changes of sleep stage. And it was confirmed by $\mathrm{T}$ test and multisamples experiments. The symbolic transfer entropy can apply into automatic sleep stage classification. By Multi-parameter analysis it could achieve a higher accuracy of sleep stage classification.
\end{abstract}

Key words: EEG; symbolic transfer entropy; sleep stage classification, $\alpha$ wave

\section{Introduction}

Sleep is a important activity of human. Sleep stage research results are important criterion for evaluating quality of human sleep. According to the performance of the electroencephalogram (EEG), with reference to the EMG signal (EMG), eye signals (EOG), sleep stages were divided into wake stages, non-rapid eye movement sleep stages (NREM) and rapid eye movement sleep stages. NREM also can be divided into I stages, II stages, III stages and IV stages. Human brain sleep EEG contains a large number of physiological and disease information which has a very important clinical value not only for providing diagnostic evidence for sleep disorders, but also for providing an effective treatment for certain sleep disorders.

Sleep EEG signals are chaotic signals which have a wide range of nonlinear dynamical characteristics [1]. The correlation dimension, Lyapunov exponent, approximate entropy were applied in the field of sleep EEG studies [2]. We know that with the deepening of sleep from the awake state, the brain activity degrees reduction of freedom which suggests that the brain cell is continuing coupling, or the original active part is continuing inacting [3]. Hence the correlation of the EEG signal enhanced.

Human body was in line with three basic characteristics of the dissipative structure. So the symbolic transfer entropy (STE) [4-6] can be use to analyze human sleep stage. Selecting one lead EEG signals from MIT-BIH Polysomnographic Database of PhysioBank, after analyzing awake stages and NREM sleep stages, it was found that the symbolic transfer entropy in the different sleep stages has different characteristic values. The symbolic transfer entropy of the $\alpha$ wave of wake stages was larger and that of NREM sleep stage was less. It can distinguish different sleep stages. The symbolic transfer entropy [4-6] as a new parameters of sleep stage can be extended to the staging of sleep in REM and NREM sleep period, and accordingly can study the structure of patients with a night's sleep. 
When normal adults are in a clear, quiet, eyes closed state, the basic rhythm of the EEG is the occipital $\alpha$ wave while at other sites it is based on a small amount of slow-wave-based $\alpha$ wave. To judge whether brain waves are normal, it is according to their age, the frequency of the EEG, volatility, on both sides of the symmetry and the number of slow-wave part of the way and the presence of pathological wave analysis. It is decisive diagnostic value for epilepsy in Khotan epileptic seizures of intermittent period because the electroencephalogram may have paroxysmal high amplitude slow wave, spikes, sharp wave, spike a slowwave integrated the so-called "painful discharge performance.

In this paper, we used STE method to analyze the $\alpha$ wave of different physiological and pathological states of epilepsy EEG signals and reached some inspired results.

\section{Theory}

\subsection{Symbolizing time series}

Assuming ECG sequence is $\mathrm{X}, \mathrm{X}$ $=\left\{x_{0}, x_{1}, x_{2}, \ldots, x_{i}, \ldots, x_{N}\right\}$, and sequence length is N.Transferring the sequence $\mathrm{X}$ into a sequence of symbols $\mathrm{S}=\left\{s_{1}, s_{2}, \ldots, s_{i}, \ldots, s_{N}\right\}, s_{i} \in A$ ( $A=0,1,2,3$ ). Specific conversion method is as follows:

Calculating differently the mean value of signals greater than zero and signals less than zero of sampled ECG signal, it is denoted by $u_{1}$ and $u_{2}$.

$$
s_{i}\left(x_{i}\right)= \begin{cases}0: & u_{1}<x_{i} \leq(1+a) u_{1} \text { or }(1+a) u_{2} \leq x_{i}<u_{2} \\ 1: & (1+a) u_{1}<x_{i}<\infty \text { or }-\infty<x_{i}<(1+a) u_{2} \\ 2: & (1-a) u_{1}<x_{i} \leq u_{1} \text { or } u_{2} \leq x_{i}<(1-a) u_{2} \\ 3: & (1-a) u_{2} \leq x_{i} \leq(1-a) u_{1}\end{cases}
$$

Where $i=1,2, \ldots, N$. And $a$ is a constant parameter. If the value $a$ is taken too large or too small, it can reduced to the loss of detail in the symbolizing original time series to symbolic sequences, and it cannot well capture dynamic information in the signals. Here we take $a=0.05$.

\subsection{Calculating the symbolic transfer entropy}

Supposing $x_{i}=x(i), y_{i}=y(i), \mathrm{i}=$ $1, \ldots, \mathrm{N}$, which represent the two observed time series. The value of $y_{i+1}$ was predicted by history related $x_{i}$ and $y_{i}$. And it was quantified the Markov bias, $\mathrm{p}\left(x_{i+1} \mid x_{i}, y_{i}\right)=\mathrm{p}\left(x_{i+1} \mid x_{i}\right), \quad \mathrm{p}$ is the transition probability density. If not haing Markov bias, it indicates $\mathrm{Y}$ having no effect on $X$. Then transfer entropy can also be seen as the permutation entropy between $\mathrm{p}\left(x_{i+1} \mid x_{i}, y_{i}\right)$ and $\mathrm{p}\left(x_{i+1} \mid x_{i}\right)$.

The transfer entropy of sequence $X$ and sequence $\mathrm{Y}$ is defined as:

$$
T_{X \rightarrow Y}=\sum p\left(x_{n+1}, x_{n}^{(k)}, y_{n}^{(l)}\right) \log \frac{p\left(x_{n+1} \mid x_{n}^{(k)}, y_{n}^{(l)}\right)}{p\left(x_{n+1} \mid x_{n}^{(k)}\right)}
$$

$x_{n} 、 y_{n}$ represent the state of $n$ time and $x_{n}^{(k)}$ is on behalf of $x_{n}, \ldots, x_{n-k+1}$, usually $k, l$ taking 1 .

Selecting two sets of data of different leads in the same time for the same individual, after a symbolized sequence were $\mathrm{S}$ and $\mathrm{J}$ respectively. Using the formula, it can be calculated the symbol transfer entropy.

$T_{S \rightarrow J}=\sum p\left(s_{n+1}, s_{n}^{(k)}, j_{n}^{(l)}\right) \log \frac{p\left(s_{n+1} \mid s_{n}^{(k)}, j_{n}^{(l)}\right)}{p\left(s_{n+1} \mid s_{n}^{(k)}\right)}$ 


\section{Data analysis}

Sleep EEG data used in this paper were from the PhysioBank the MIT-BIH Polysomnographic Database. Records in the Database is a multi-parameter sleep data, including EEG, ECG, EOG, EMG etc. The data sampling frequency is of $250 \mathrm{~Hz}$. The paper used one lead EEG signals of multiparameter sleep data from subject slp41 (C4-A1) whose wake stage and NREM I sleep stage signal was extracted. Each stage was of 7500 sampling points. The sleep process is continuous, so the 7500 point may not entirely belong to a sleep stage and we used 2000 sampling points between $2500-5000$ points.

When multisample confirming experi mental laws, it was randomly selected three samples which were taken in this experiment were slp41, slp48, slp59. The EEG leads were slp41 (C4-A1), slp48 (C3-O1), slp59 (C3-O1) respectively. slp41 (C4-A1) was on behalf of the receiver 41 whose EEG was obtained through the C4-A1. Similarly, slp48 (C3O1), slp59 (C3-O1) showed that 48th and 59th subjects' EEG were taken from leads C3-O1.

It was randomly extracted 5 wake stage signals and 5 NREM I stage signals from subjects slp41 with data length 2000 . The mean and variance of symbolic transfer entropy for these signals when at $n=4$ were calculated. The experimental results were shown in Tab. 1.

Table 1: slp59 sample,for example,the statistics of the different sampling points the wake (w) and NREM I sleep stage(i)

\begin{tabular}{|c|c|c|c|c|c|c|c|c|c|c|}
\hline \multirow{2}{*}{$\begin{array}{c}\text { sampling } \\
\text { points }\end{array}$} & \multicolumn{2}{|c|}{500} & \multicolumn{2}{c|}{1000} & \multicolumn{2}{c|}{2000} & \multicolumn{2}{c|}{3000} & \multicolumn{2}{c|}{4000} \\
\cline { 2 - 11 } & $\mathbf{W}$ & $\mathbf{i}$ & $\mathbf{W}$ & $\mathbf{i}$ & $\mathbf{W}$ & $\mathbf{i}$ & $\mathbf{W}$ & $\mathbf{i}$ & $\mathbf{W}$ & $\mathbf{i}$ \\
\hline 1 & 0.2119 & 0.1240 & 0.2312 & 0.1101 & 0.0686 & 0.0685 & 0.0370 & 0.0417 & 0.0282 & 0.0326 \\
\hline 2 & 02230 & 0.1232 & 0.1642 & 0.1061 & 0.0681 & 0.0559 & 0.0415 & 0.0427 & 0.0363 & 0.0298 \\
\hline 3 & 0.1960 & 0.1177 & 0.2033 & 0.1147 & 0.0789 & 0.0634 & 0.0486 & 0.0474 & 0.0292 & 0.0288 \\
\hline 4 & 0.2102 & 0.1180 & 0.2000 & 0.1322 & 0.0487 & 0.0651 & 0.0415 & 0.0372 & 0.0308 & 0.0297 \\
\hline 5 & 02387 & 0.1337 & 0.1657 & 0.1224 & 0.0547 & 0.0506 & 0.0442 & 0.0405 & 0.0364 & 0.0222 \\
\hline 6 & 0.1775 & 0.1122 & 0.2237 & 0.1048 & 0.0749 & 0.0598 & 0.0459 & 0.0471 & 0.0323 & 0.0297 \\
\hline 7 & 0.1994 & 0.1178 & 0.2108 & 0.0993 & 0.0567 & 0.0531 & 0.0471 & 0.0357 & 0.0310 & 0.0325 \\
\hline 8 & 0.2468 & 0.1387 & 0.2003 & 0.1014 & 0.0648 & 0.0746 & 0.0485 & 0.0448 & 0.0302 & 0.0321 \\
\hline 9 & 0.2299 & 0.1304 & 0.1770 & 0.1137 & 0.0626 & 0.0490 & 0.0509 & 0.0371 & 0.0353 & 0.0294 \\
\hline 10 & 0.2300 & 0.1247 & 0.2041 & 0.1044 & 0.0628 & 0.0408 & 0.0424 & 0.0388 & 0.0303 & 0.0373 \\
\hline mean & 0.2163 & 0.1240 & 0.1986 & 0.1109 & 0.0641 & 0.0581 & 0.0448 & 0.0414 & 0.0320 & 0.0304 \\
\hline std & 0.0214 & 0.0082 & 0.0228 & 0.0102 & 0.0092 & 0.0102 & 0.0042 & 0.0040 & 0.0030 & 0.0038 \\
\hline
\end{tabular}

Further, we analyzed other different individual data, respectively. The analyzed data were 500 sampling points. Af-

ter the mean-variance table was obtained, it was given the $\mathrm{T}$ test.

Table 2: Average of STE values for different individual with 500 sampling points

\begin{tabular}{|l|c|c|c|c|c|c|c|c|}
\hline datas & Slp590 & Slp480 & Slp410 & Slp450 & Slp591 & Slp481 & Slp411 & Slp451 \\
\hline Mean w & 0.2163 & 0.1809 & 0.1990 & 0.1941 & 0.2223 & 0.1537 & 0.1429 & 0.1736 \\
\hline Mean i & 0.1986 & 0.0951 & 0.1347 & 0.1424 & 0.2155 & 0.1119 & 0.1163 & 0.1485 \\
\hline
\end{tabular}


The following is the $\mathrm{T}$ test results.

Paired Samples Statistics

\begin{tabular}{|ll|l|r|r|c|}
\hline & & Mean & $\mathrm{N}$ & Std. Deviation & $\begin{array}{c}\text { Std. Error } \\
\text { Mean }\end{array}$ \\
\hline Pair1 & Wake stage & .109086 & 7 & .0345688 & .0130658 \\
& NREM1 stage & .086257 & 7 & .0346830 & .0131090 \\
\hline
\end{tabular}

Paired Samples Correlations

\begin{tabular}{|l|r|r|r|}
\hline & $\mathrm{N}$ & Correlation & \multicolumn{1}{c|}{ Sig. } \\
\hline $\begin{array}{l}\text { Pair1 } \\
\begin{array}{l}\text { Wake stage \& NREM1 } \\
\text { stage }\end{array}\end{array}$ & 7 & .825 & .022 \\
\hline
\end{tabular}

Paired Samples Test

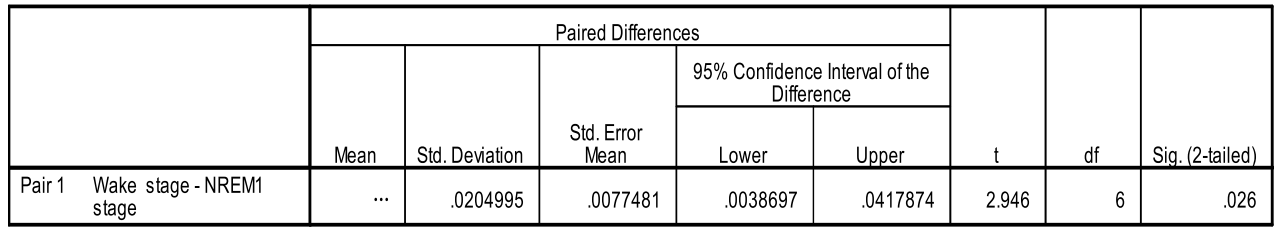

According to Tab. 2, the mean and variance of the symbolic transfer entropy for these signals were shown in Figure 1.

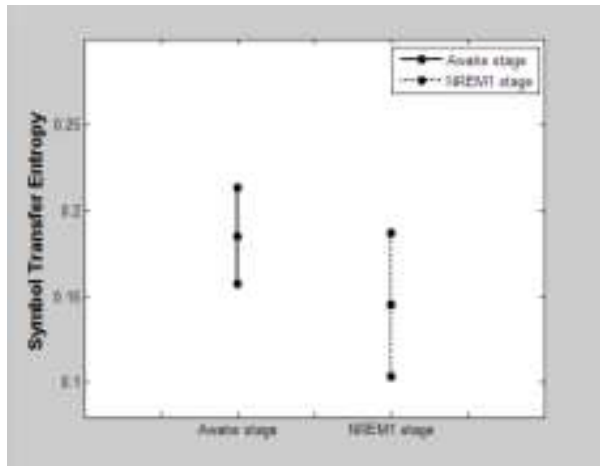

Figure 1: STE range in two sleep stages with 500 sampling points.

\section{Discussion}

Reflect the relevance of the two time series or degree of confusion STE STE larger the value, the greater uncertainty, the sequence of a large degree of confusion. Instead, STE smaller the value, the smaller uncertainty, the degree of serial correlation.sober period of the brain from the outside world to accept more nerve cell activity randomness brain greater freedom of movement, the brain is more active, the two time series degree of confusion, STE value. sleep into NREM sleep stage.brain, though they do not stop their activities, but when muscle relaxa- tion, nerve reflexes, degrees of freedom to reduce brain activity, brain cell coupling, or the original active part inactivation EEG degree of confusion decreases STE value decreasesseen that the experimental results and the theoretical analysis is consistent.

\section{Conclusions}

Preliminary exploration of this paper as an the STE application in sleep EEG analysis to distinguish between waking and NREM sleep stage I, and prove the feasibility of the method. STE-based sleep staging method can be further extended to Phase II of NREM sleep,distinguish phase III, IV and REM period. mentioned in this article STE, a quantitative parameters of the relevance of the two time series, this algorithm reduces the coordination requirements between the parameters calculated easily and quickly, suitable for real-time processing,also reduce the sensitivity to noise, certain anti-jamming capability. these features greatly facilitated the transfer entropy time series analysis.

Sleep is an extremely complex and dynamic process, clinical, are based on a multi-parameter by experienced doctors artificial sleep staging has a close relationship. Proposed in this paper the STE and sleep stage, can extract various stag- 
ing eigenvalue, very good expression changes in the sleep state, a new judge for the sleep automation installments research increased.

\section{Acknowledgements}

This work was supported by the National Natural Science Foundation of China (Grant Nos. 61271082, 61201029, 61102094), the Natural Science Foundation of Jiangsu Province (Grant Nos. BK2011759, BK2011565) and Foundation of Nanjing University of Posts and Telecommunications (JG03212JX02, JG03210JX19, 2011XSG11).

\section{References}

[1] Q.F. Meng, W.D. Zhou, Y.H. Chen, Y.H. Peng, "The feature extraction of epileptic EEG signals based on nonlinear prediction," acta phys. sin., fdpp.123-130, 2010. (in Chi nese)

[2] L.L. Zhao, Z.Q. Liang, W.Q. Wu, G.S. Hu, "Changes of EEG Correlation Dimensions in Epilepsy after Biofeedback Training," Chin. J. Biomed. Eng., pp.71-76,85, 2010. (in Chinese )

[3] Q.L. Ma, C.H. Bian, J. Wang, "Scaling analysis on electroencephalagram and its application to sleep-staging, " acta phys. sin., pp.4480-4484, 2010. (in Chinese)

[4] T. Schreiber, "Measuring information transfer," Phys. Rev. Lett., pp.461-464, 2000.

[5] M. Staniek, K. Lehnertz, "Symbolic transfer entropy," Phys. Rev. Lett., pp.158101, 2008.

[6] E. Roldan, M.R.J. Parrondo, "Estimating dissipation from single stationary trajectories," Phys. Rev. Lett., pp.150607, 2010. 